


\section{Zum Geleit!}

Das "Handbuch der Wirtschafts- und Sozialwissenschaften: sucht in abgeschlossenen „Einzelbänden “, also monographiscl, der weitgesteckten Aufgabe gerecht zu werden, Lehrern und Lernenden, Theoretikern und Praktikern des Wirtschafts- und Gesellschaftslebens Beratung, Bereicherung, Vertiefung, aber auch Stoff zu eigner kritischer Stellungnahme zu bieten. Die Umwälzungen auf fast allen Gebieten des öffentlichen Lebens, zumeist dauernder Art, vielfach im FluB, aber manchmal wohl schon dem AbschluB nahe, machen an sich bereits ein Unternehmen wie das gegenwärtige verständlich; denn nicht immer wird es möglich sein, die unter ganz anderen Voraussetzangen verfaßten früheren Kompendien durch Neuauflagen der Zeit und ihren Anforderungen gemäß zu gestalten. Daß nach dem heutigen Stand der Wissenschaft nur arbeitsteiliges Zusammenwirken von Spezialisten die notwendige Sachkunde gewährleistet, die dann erst die Zusammenpressung auf nicht allzugroßen Raum ermöglicht, dürfte keinem Zweifel begegnen, ebensowenig, da $B$ unsere Arbeitsgemeinschaft den Sachkenner zum Worie kommen läßt. Fraglich könnte eher sein, ob es möglich war, so viele verschiedeneStandpunkte, die oft auch ungleiche Methoden enthalten, unter einer Generalidee zusammenzuführen. Das war aber auch nicht in dem Sinne beabsichtigt, daß dem einzelnen Mitarbeiter ein - heute schlechterdings unmöglicher - Verzicht auf selbständige Stellungnahme auferlegt wäre. Eher waren die Herausgeber bemüht, verschiedene, ja gegensätzliche Auffassungen zum Wort kommen zu lassen, die sich aber doch in dem Streben nach theoretischer Stoiffurchdringung und praktischer Stoffauswirkung begegnen; die Verfasser dieser Bände treten selbst wertend an die wirtschaftlichen und sozialen Probleme heran und geben ein bestimmtes Bild von dem, was für den Wiederaufbau der deutschen nationalen Wirtschaft und der staatlichen Gesellschaft entscheidet.

Das Gebiet des "Handbuchs" umfaßt, wie die folgende Übersicht zeigt, Gegenstände, đie früher den "Staatswissenschaften " zurechneten, deren systematischer Ort heute aber zweifellos in den "Sozialwissenschaften " liegt; aus der ,Wirtschaftslehre“ kommen die Disziplinen der Privat-(Betriebs-) Wirtschaftslehre, ihrer jetzigen Bedeutung gemäB, ausgiebig zum Wort; neben den als solche unmittelbar deutlichen reinen Spezialbänden finden sich zusammenfassende Darstellungen mit stark betonten methodologischen und systematischen Absichten; daß aber die sogenannte "Praktische oder spezielle Nationalökonomie* nicht als solche, sondern in ihre Teilgebiete aufgelöst erscheint, wird kein Kenner verurteilen.

Der Anschluß an die bisherigen Systeme und Auffassungen ist hier ein engerer, dort ein weiterer; in beiden Fällen müssen ganz neue Wege gegangen werden. Alle Beteiligten sind sich der Verantwortung bewußt, die ein Lehrund Handbuch heute hat, bei dem ungeheuren Zustrom zu den Wirtschafts- und Sozialwissenschaften und angesichts der Notwendigkeit, daß die Theorie auch in derPraxis wieder Beachtung gewinnt; die gestörten Verhältnisse in der Gegenwart können ja nur in engster Zusammenarbeit von Theorie und Praxis geändert und hôffentlicl gebessert werden.

Ob sie nun in der Hand des akademischen Lehrers diesen entlasten, ob sie als Einführung oder Repetitorium den Studierenden unterstützen; ob sie außerhalb der eigentlichen Hochschulen, für staatsbürgerliche Erziehung werben; ob sie dem Praktiker für das Labyrinth der Einzelfragen des täglichen Lebens als Führer dienen: in jedem Falle wollen die zum "Handbuch" vereinigten "Einzelbände " ein treues Abbild der Gegenwart sein; ihrer Strömungen, Befürchtungen, Hoffnungen, Aussichten, und vor allem: ihrer starken theoretischen Selbstbestimmung.

$$
\text { Die Herausgeber - Die Verlagsbuchhandlung }
$$





\section{Handbuch \\ der \\ Wirtschafts- und Sozialwissenschaften}

i n E i n z e l b ä n d e n.

Unter Mitwirkung von

GUSTAV AUBIN, Halle, GEORG VON EBERT, Nürnberg, ALEXANDER ELSTER, Berlin, WILHELM GERLOFF, Frankfurt a. M., FR. HOFFMANN, Rostock, RUDOLF MEERWARTH, Berlin, PAUL MOLDENHAUER, Köln, OTTO MOST, Duisburg-Ruhrort, MAX MUSS, Leipzig,WILHELM RIEGER,Nürnberg, MAX RUMPF, Mannheim, AUGUST SKALWEIT, Kiel, BRUNO SKALWEIT, Königsberg, FRIT Z TERHALLE, Hamburg, WALDEMAR ZIMMERMANN, Hamburg

herausgegeben von

ADOLF GÜNTHER und GERHARD KESSLER Innsbruck Jena.

\section{Siebzehnter Band.}

August Skalweit: Agrarpolitik.

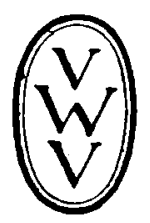

Berlin und Leipzig 1923.

Walterde Gruyter\& Co.

vormals G.J.Grschen'sche Verlagshandlung - J. Guttentag, Verlagsbuchhandiung - Georg Reimer - Karl J. Trübner - Veit \& Comp. 


\section{Agrarpolitik}

von

\section{Dr. August Skalweit,}

Ordl. Professor der Volkswirtschaftslehre an der Universität Kiel.

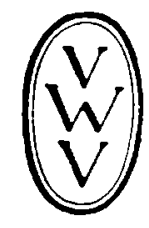

Berlin und Leipzig 1923.

Walterde Gruyter \& Co.

vormals G.J.Göschen sche Verlagshandlung - J. Guttentag, Verlagsbuchhandlung - Georg Reimer - Karl J. Trübner - Veit \& Comp. 
RoBberg'sche Buchdruckerei, Lejpzig. 
MAX SERING

gewidmet 
